\title{
Correction to: Improving Control Performance of Unmanned Aerial Vehicles through Shared Experience
}

\author{
Peter Travis Jardine ${ }^{1} \cdot$ Sidney Givigi ${ }^{2}$ (D)
}

Published online: 22 January 2022

C) Springer Nature B.V. 2022

Correction to: J Intell Robot Syst (2021) 102: 68.

http://doi.org/10.1007/s10846-021-01387-1

In this article ref. 23 was incorrect and should have been "Petrović VM: Artificial intelligence and virtual worlds- toward human-level ai agents. IEEE Access 6, 39,976-39,988 (2018)". The original article has been corrected.

Publisher's Note Springer Nature remains neutral with regard to jurisdictional claims in published maps and institutional affiliations.

The original article can be found online at https://doi.org/10.1007/ s10846-021-01387-1.

Sidney Givigi

sidney.givigi@queensu.ca

Peter Travis Jardine

peter.jardine@rmc.ca

1 Department of Electrical and Computer Engineering, Royal Military College of Canada, Kingston, Canada

2 School of Computing, Queen's University, Kingston, Canada 